JASC 12-1-6

\title{
File Modification Pattern Detection Mechanism Using File Similarity Information
}

\author{
Ho Min Jung, Yong Woong Ko
}

Dept. of Computer Engineering, Hallym University, Korea

\begin{abstract}
In a storage system, the performance of data deduplication can be increased if we consider the file modification pattern. For example, if a file is modified at the end of file region then fixed-length chunking algorithm superior to variable-length chunking. Therefore, it is important to predict in which location of a file is modified between files. In this paper, the essential idea is to exploit an efficient file pattern checking scheme that can be used for data deduplication system. The file modification pattern can be used for elaborating data deduplication system for selecting deduplication algorithm. Experiment result shows that the proposed system can predict file modification region with high probability.
\end{abstract}

Key words : Deduplication, File modification, File pattern, Fixed-length Chunking, Variable-length Chunking

\section{INTRODUCTION}

Data deduplication is widely used in a storage system as a means of reducing the storage size and providing low bandwidth file transferring mechanism[1]. Data deduplication can eliminate redundant data by retaining only one copy of the data and reduce the required storage capacity.

The primary goal of this work is to provide a scheme that allows for efficient data deduplication by utilizing file modification pattern using file similarity information. In a backup system or versioning file system, many files have duplicated data blocks between files because previous version of file may be modified by adding additional data at the end of file position. In this case, we can easily eliminate duplicated information using fixed-length chunking algorithm by simply dividing data file with fixed size chunk and by checking duplication using hash comparison. However, if the front part of the file is modified, fixed-length chunking will show worst performance result for its inherent problem known as "byte shifting problem". Therefore, to predict file modification pattern is very important in a deduplication system.

Manuscript received May. 7, 2012; revised May.23, 2012

†Corresponding Author: yuko@hallym.ac.kr

Tel: +82-33-248-2329, Fax: +82-33-242-2524, Hallym Univ.

*Dept. of Computer Engineering, Hallym Univ., Korea
To address these problems, we propose a deduplication system that addresses these issues by adapting dynamic policy changing algorithm considering file modification pattern. The key idea is to exploit file similarity information for predicting file modification pattern. In the proposed system, each file has a file similarity information that composed of representative hash keys and location offset in a file. By utilizing the file similarity information, we can exactly predict in which section of file is modified.

The rest of this paper is organized as follows. In Section 2, we describe related works and In Section 3, we explain the design principle of proposed system and implementation details for File deduplication system. In Section 4, we show performance evaluation result of file pattern. Finally, we conclude and discuss future research plan.

\section{RELATED WORKS}

In content-defined chunking or variable-length chunking[2], each block is partitioned using special hash values called anchor. This scheme can prevent the data shifting problem of fixed-length chunking which is a fast deduplication approach. One of the well-known content-defined chunking algorithms is LBFS[3], a network file system designed for low bandwidth networks. LBFS eliminates data duplication between files or versions of the same files in order to reduce network bandwidth. LBFS avoids sending data blocks over a network when the same data can already be found in the 
server's file system or the client's cache. With this approach, LBFS can achieve up to two orders of magnitude in bandwidth utilization compared to traditional network file systems. Delta encoding stores data in the form of differences between sequential data[4]. Many backup systems widely adopt this scheme in order to give their users previous versions of the same file from previous backups. Delta encoding reduces costs in the amount of data that has to be stored as differing versions, as well as the costs in the uploading of each file that has been updated.

\section{SYSTEM DESIGN AND IMPLEMENTATION}

In this paper, we apply file similarity concept for searching file modification position. File similarity search concept is widely used in data deduplication system and data processing system area. To check file similarity, we first extract sample hash keys from a file. For data block hash key, Rabin hash function is very famous and efficient algorithm. It calculates hash key from a file with fixed size and it generates another hash key by shifting one byte repeatedly. If Rabin hash calculation is finished, we choose the maximum or minimum hash keys. These key values are used for sample hash key for a file. And we can easily compare the file similarity between two files. When A file and B file have duplicated Rabin hash keys, this means that the files have duplicated data blocks.

In previous result[5], we proposed the file modification pattern algorithm using file similarity, however, the result has limitation on supporting several modification point in a file. Therefore, we enhanced the algorithm as shows in Figure 1.

The main purpose of file modification pattern search algorithm is to predict the relationship between two files using file similarity information. For Head section and End section algorithm, we described detailed explanation in [5]. Here, we only describe enhanced file modification search algorithm.

In Figure 1, elements in each array (Array1, Array2) contains two tuples: file hash key (Array.hash) and file offset (Array.offset) value that is a block start location in a file. Algorithm compares hash key elements in each array. cnt means the number of block region which contains non-duplicated data. shift value contains the difference between two location. First, if two hash keys extracted from Array1 and Array2 are equal, IsEqual is set to TRUE and stores location difference into shift value. In Figure 1, if shift value is greater than beforeshift then it means new data is added between the same hash array. In this case, we increase addition value. If shift value is less than beforeshift then it means that previous data is deleted and we increase deletion value. When there are data insertion or deletion, we have to perform data deduplication using MidSection function.

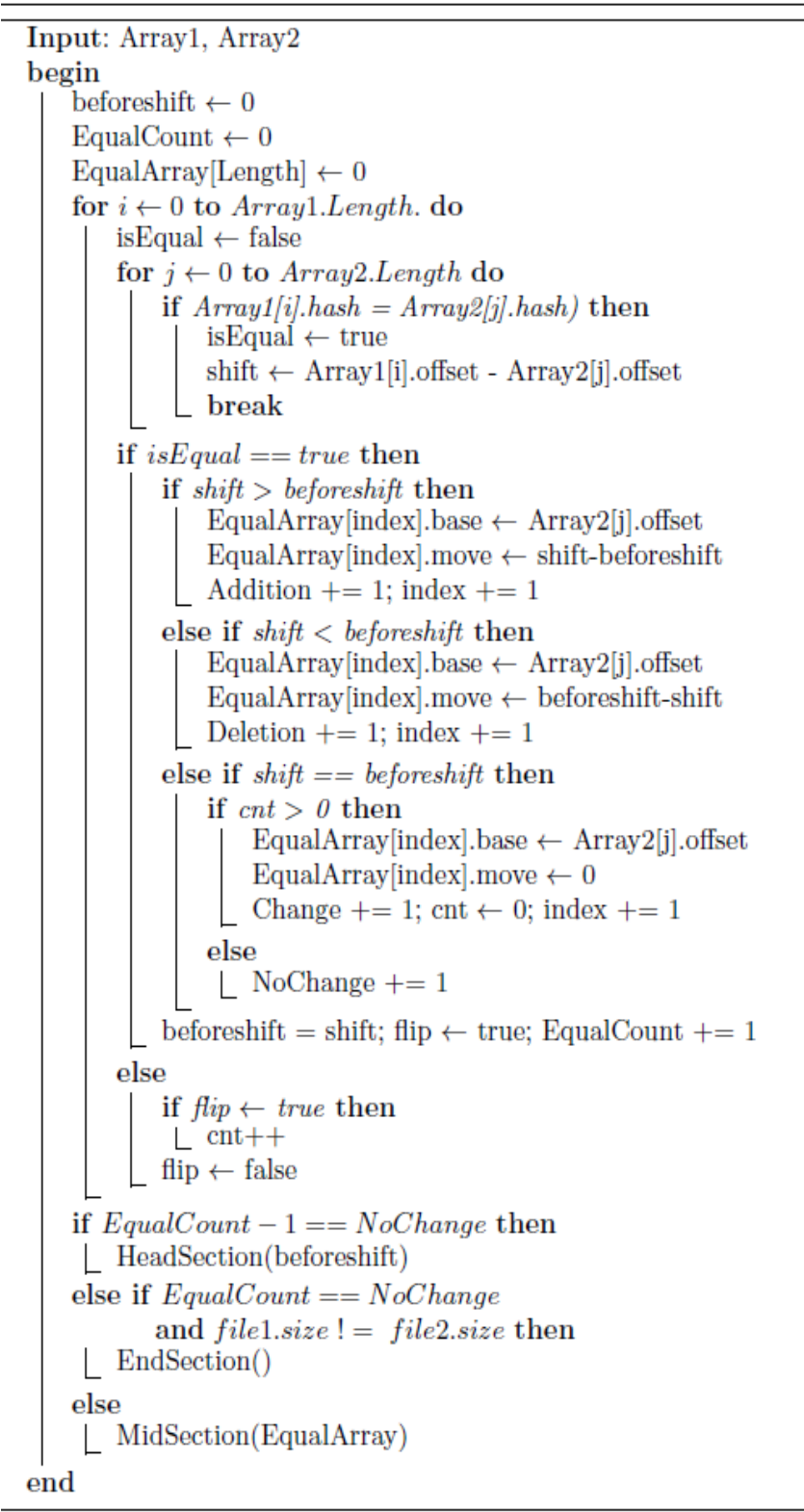

Fig. 1. File modification pattern search algorithm.

In [5], we explain how HeadSection() and EndSection() function are work. HeadSection() is a deduplication algorithm for a file that has modified section in front of the file. EndSection() function is processed in case that a file has modified section at the end of the file. In this case, almost all section of the front region is identical therefore the proposed system begins deduplication process immediately until non-duplicated region is found. In this paper, we added MidSection() for data deduplication when there is file modification in middle section of a file. When there are file modification, MidSection() function applies fixed-length chunking approach by using EqualArray parameter. EqualArray represents file modification status using base and 
move value. The base value means the difference of the same hash key position between two files. The move value means how much the data is skewed. By using the base and move value, we can use fixed-length chunking approach until there is non-duplicated block.

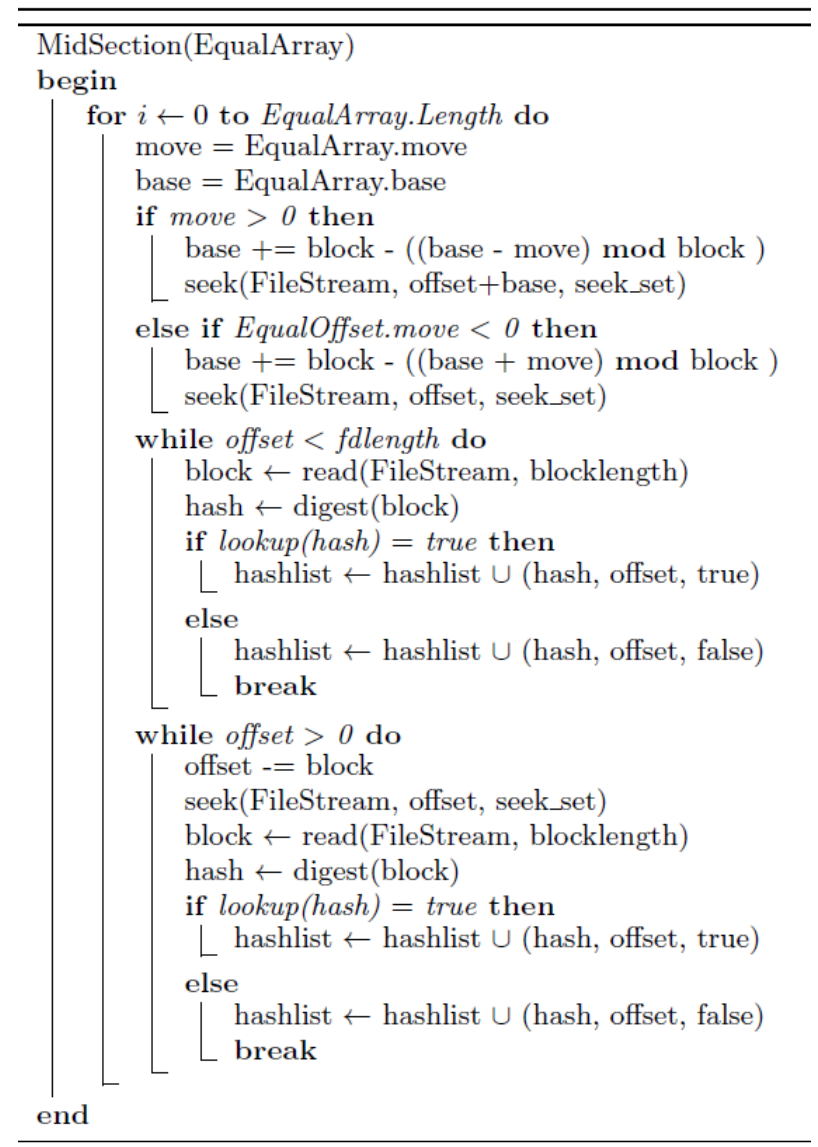

Fig. 2. Data deduplication using MidSection() function

\section{RESULTS AND DISCUSSION}

To perform comprehensive analysis on the proposed algorithm, we implemented the client and the server on the platform that consists of $3 \mathrm{GHz}$ Pentium 4 Processor, WD-1600JS hard disk and 100Mbps network.

Figure 3 demonstrates the impact of the hash key elements in the file similarity information. The Head and Tail means that the file is modified at the beginning section and at the tail section, respectively. Mid means that the file is modified at the middle section. Especially, if there are many modification points then we used Mid(Many) or we used Mid(Few).

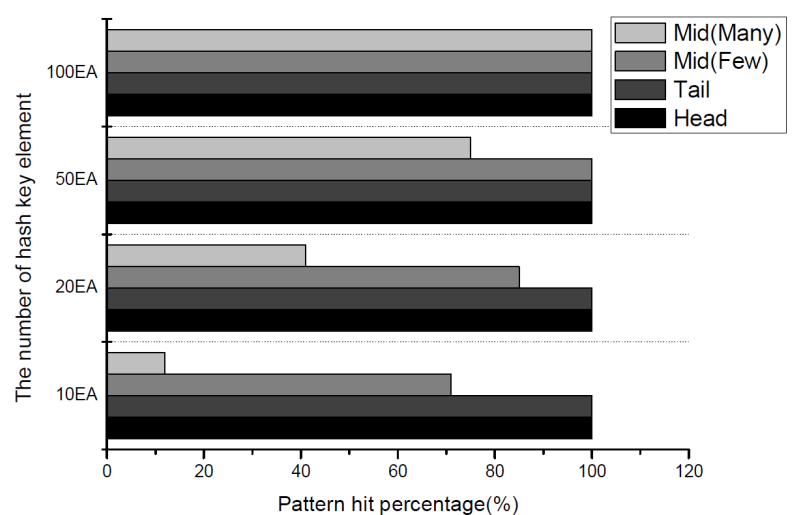

Fig. 3. Evaluation result for pattern hit while varying the number of hash key elements.

We evaluated file modification pattern hit percentage varying the number of hash key elements from 10 up to 100 . We found that file modifications in the Head Section and End Section are perfectly examined with small number of hash key elements. However, modifications in the Mid Section needs many hash key elements for high pattern hit. The evaluation results explain that the proposed scheme efficiently performs fixed-length chunking for Head Section and End Section with low overhead.

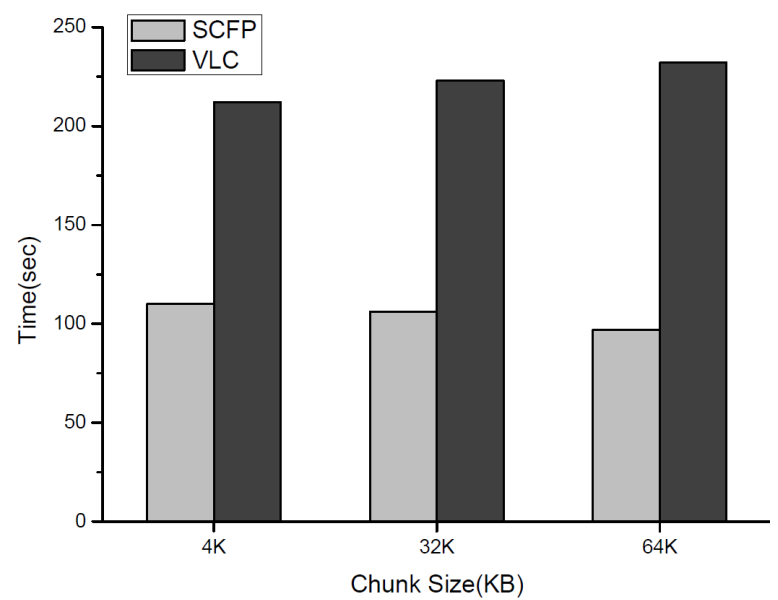

Fig. 4. Evaluation result for processing time: SCFP vs. VLC

In this experiment, we compared the processing time of data deduplication between SCFP(Static Chunking using File Pattern) and VLC(Variable-Length Chunking) varying chunk size. For realistic experiment, we patched 10 data blocks to 2.3Gbyte data file. The overall processing time of SCFP distributed between 97 to 110 seconds. However, the processing time of VLC is roughly 2 times longer than SCFP. 


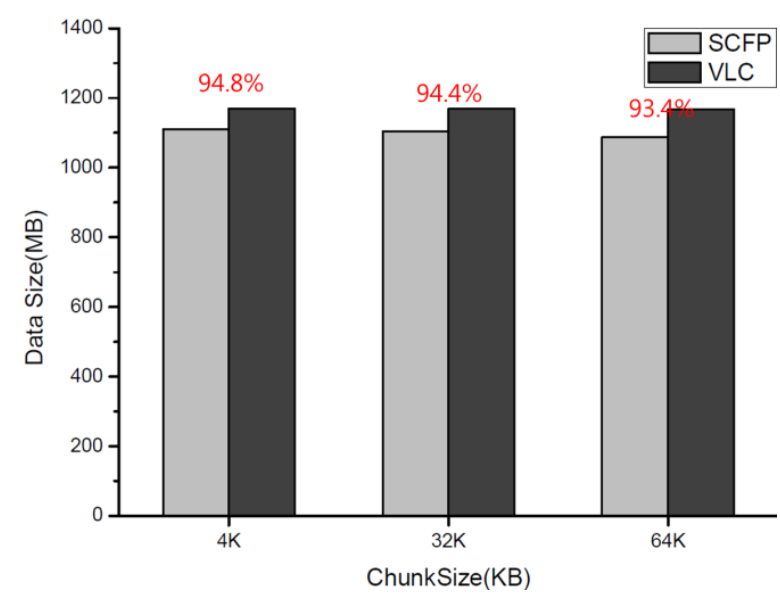

Fig. 5. Evaluation result for space reduction : SCFP vs. VLC

Figure 5 shows the space reduction result for the proposed system varying chunk size. The evaluation result shows that the proposed system(SCFP) can achieve 94\% of space reduction capability compared with VLC. However, as can be seen Figure 4, the processing time of SCFP is very fast compared with VLC, so we believe that the proposed system is superior to VLC approach.

\section{CONCLUSION}

This paper presents an enhanced storage system utilizing file modification pattern to achieve high performance deduplication throughput and capacity. the proposed deduplication system provides dynamic policy changing algorithm considering file modification pattern using file similarity information. We have found that using file modification pattern is superior to traditional data deduplication. In experiment result, with few hash keys from file similarity information, we can predict in which section is modified between files. Furthermore, the proposed system perfectly predicts regardless of patch size for file modification in front of a file or at the end section of a file.

However, several issues remain open, First, our work has limitations on supporting simple file modification pattern that covers file modification at the end of a file or front part of a file. If there happens several modifications on a file, data deduplication algorithm have to consider this case. Finally, we plan to build a massive deduplication system with huge number of files. In this case, handling file similarity information needs more elaborated scheme.

\section{ACKNOWLEDGMENT}

This research was financially supported by Basic Science Research Program through the National Research Foundation of Korea(NRF) funded by the Ministry of Education, Science and Technology(2010-0016143)

\section{REFERENCES}

[1] Quinlan, S. and Dorward, S, "Venti: a new approach to archival storage," in Proc 1st USENIX conference on File and storage technologies, pp. 7-7. ,2002.

[2] Eshghi, K. and Tang, H.K., "A framework for analyzing and improving content-based chunking algorithms," Hewlett-Packard Labs Technical Report TR, vol. 30,2005 .

[3] Muthitacharoen, A., Chen, B. and Mazieres, D, "A low-bandwidth network file system," $A C M$ SIGOPS Operating Systems Review 35, pp. 174-187, 2001.

[4] Douglis, F. and Iyengar, A, "Application-specific delta-encoding via resemblance detection," In Proceedings of the USENIX Annual Technical Conference, pp. 1-23. 2003.

[5] Ho Min Jung, Sang Yong Park, Jeong Gun Lee and Young Woong Ko, "Efficient Data Deduplication System Considering File Modification Pattern,",International Journal of Security and Its Applications, APR. 2012.

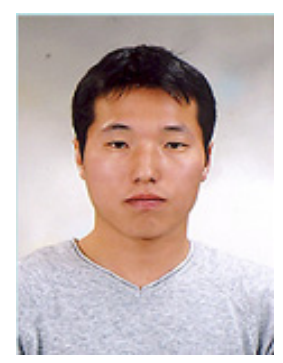

operating systems.
Ho Min Jung received the B.S., M.S. and Ph.D. degrees in Department of Computer Engineering from Hallym University, Korea, in 2006, 2008 and 2012, respectively. Since 2012, he has been with Department of Computer Engineering, Hallym University, where he is currently a Research Associate. His research interests are in the areas of Data deduplication, storage system and

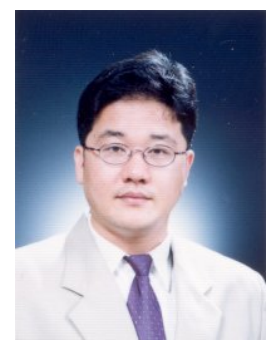

Young Woong Ko received the B.S., M.S. and Ph.D. degrees in Department of Computer Engineering from Korea University, Korea, in 1997, 1999 and 2003 respectively. Since 2003, he has been with the Department of Computer Engineering, Faculty of Engineering, Hallym University, where he is currently a professor. His research interests are in the areas of storage system, operating systems, and embedded systems. 